\title{
GALLSTONE ILEUS: A CASE REPORT
}

\author{
DANIEL CAPDEVILLE TANURE, JANSEN CHERFANI TANURE, FRANÇOISE RIBEIRO DOFFÉMOND, SAMIR ALMEIDA BORGES, \\ CÉZAR LIMA MOTA
}

\begin{abstract}
The authors present a case of mechanical bowel obstruction, uncommon, due to the passage of a gallstone through a cholecystoenteric fistula. The clinical presentation, the pathophysiology, the diagnosis and treatment possibilities are commented on. The diagnosis requires high clinical suspicion, since symptoms are usually nonspecific and complementary exams don't always help in the etiopathogenic diagnosis.
\end{abstract}

KEYWORDS: GALLSTONE ILEUS; BILIOENTERIC FISTULA; ULTRASOUND.

\section{INTRODUCTION}

Gallstone ileus is an obstruction of the intestinal transit caused by the migration of a large gallstone to the intestinal lumen, which in $70 \%$ of cases occurs through a cholecystoduodenal fistula ${ }^{1}$. This is a rare cause (1-4\%) of intestinal obstruction, although it may correspond to up to $25 \%$ of non-strangulated obstructions in patients over 65 years ${ }^{2}$. The mean age of presentation is 74 years and the mean diagnosis delay is 3.5 days $^{3}$.

Biliary ileum-related mortality ranged from $5.5-30 \%{ }^{4-6}$ depending on the site of obstruction and surgical approach.

Abdominal computed tomography (CT) with contrast is the gold standard imaging test 7 , with sensitivity, accuracy and specificity of $93 \%, 100 \%$ and $99 \%$, respectively 8 for the diagnosis of gallstone ileus.

In this case, however, the suspicion of the gallstone ileus was due to the ultrasound findings of evident perforation of the gallbladder wall, echogenic formation with posterior shadow suggestive of calculus in the proximal intestinal loop, and clear retrograde mobility of the intestinal liquid content to the gallbladder lumen during peristalsis.

\section{CASE REPORT}

A 78-year-old female patient sought medical attention due to acute discomfort in her upper abdomen. On examination she had a globose abdomen, slightly painful on palpation. She received symptomatic treatment returning for three days in a row.

On the third day, she reported worsening of abdominal discomfort, as well as onset of bloating and vomiting. She was evaluated by the surgeon on duty and had a flaccid, painless abdomen, with no signs of peritoneal irritation.

She was referred for a total abdominal ultrasound, which showed the following findings:
- Atypically shaped gallbladder, without evident content, with irregular walls and an area suggestive of communication $(1.35 \mathrm{~cm})$ with a thin loop, in whose light an arched hyperechoic image is observed, with an intense posterior acoustic shadow, suggestive of calculus, measuring ${ }^{3}, 2 \mathrm{~cm}$ in diameter - appearance suggestive of cholecystocolonic fistula (figure 1).

Presence of distended slender bowel loops $(4.0 \mathrm{~cm})$, with evident conniving valves and mobile liquid content, with increased peristalsis and retrograde flow, especially in the left hemi-abdomen (figure 2).

Observation of anechoic laminar areas between the loops and in the hepatorenal space, suggestive of free fluid.

- Normal liver and bile ducts.

- Conclusion of the report: echographic signs suggestive of intestinal obstruction (gallstone ileus).

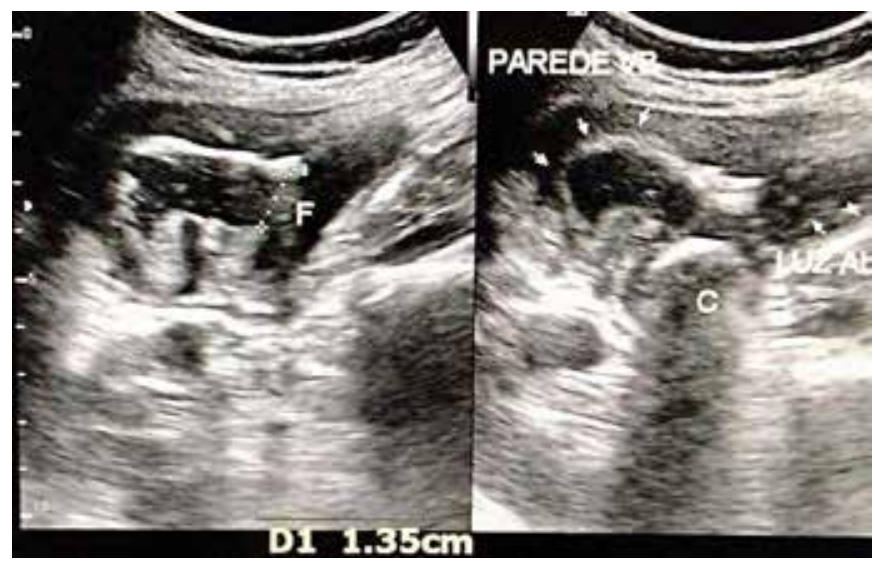

Figure 1: Left: minimum parietal opening of $1.35 \mathrm{~cm}$, communicating with the thin loop lumen (cholecystoenteric fistula). Right: calculus (C) and characteristic posterior acoustic shadowing. Arrowheads: anterior wall of the gallbladder (VB).
1. Hospital São Vicente de Paulo, Araçuaí, MG, Brasil
MAILING ADDRESS

DANIEL CAPDEVILLE TANURE

Email: danielcapdeville@hotmail.com 


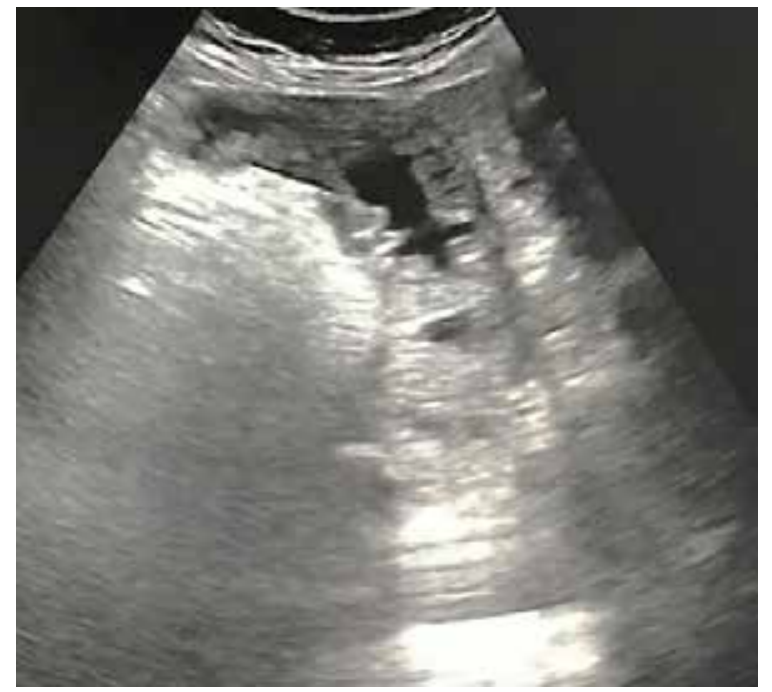

Figure 2: Sentinel loop segment.

The patient was admitted for follow-up by the surgical team.

Initial laboratory tests showed no changes. Upper digestive endoscopy (UDE) was performed, which showed:

- Esophagus: normal

- Stomach: large amount of bilious secretion, with normal endoscopic appearance of mucosa and mucosal relief of the fundus and body.

- Duodenum: bulb deformed by the presence of a deep ulcer in the posterior wall, with an irregular and necrotic background. Regurgitation of bilious intestinal material. Second portion without macroscopic changes, showing the regurgitation of bilious material in large volume (figure 3 ).

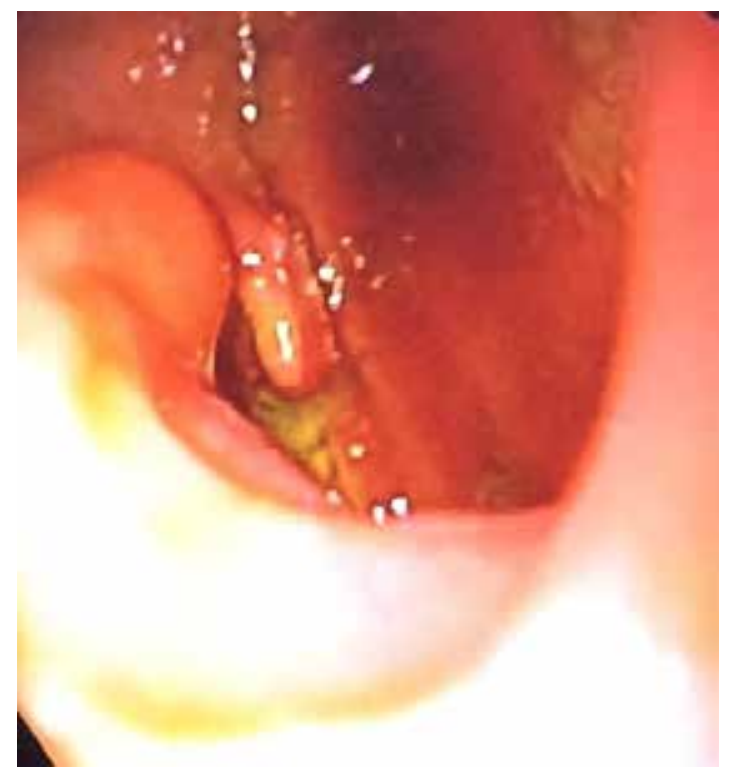

Figure 3: Cholecystoenteric fistula and biliary reflux seen in the UDE.
Expectant conduct was initially adopted, due to the patient's clinical stability. Abdominal CT was requested for follow-up, which showed:

- Marked distension of thin loops up to $4.5 \mathrm{~cm}$ in caliber and abrupt tapering in the midline of the hypogastrium, findings suggestive of intestinal subocclusion, without identification of the obstructive factor.

- Signs of hepatic aerobilia and unidentified gallbladder.

On the 6th day of hospitalization, she presented with vomiting and cramping abdominal pain. On the 8th day, she evolved with prostration, darkened vomiting in large volume and worsening of abdominal distension and pain, still without signs of peritoneal irritation.

She underwent exploratory laparotomy on the 9th day of hospitalization. Upon inspection of the cavity, obstruction of the small intestine near the terminal ileus was observed, in a place of abrupt narrowing. Through the enterotomy, two gallstones were removed (figure 4-6), with subsequent enterorrhaphy, revision of the cavity and closing by planes.

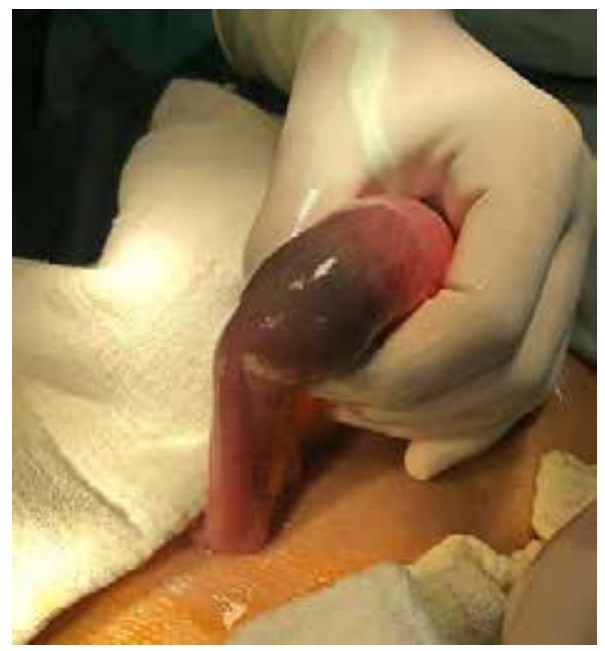

Figure 4: Intraluminal calculus

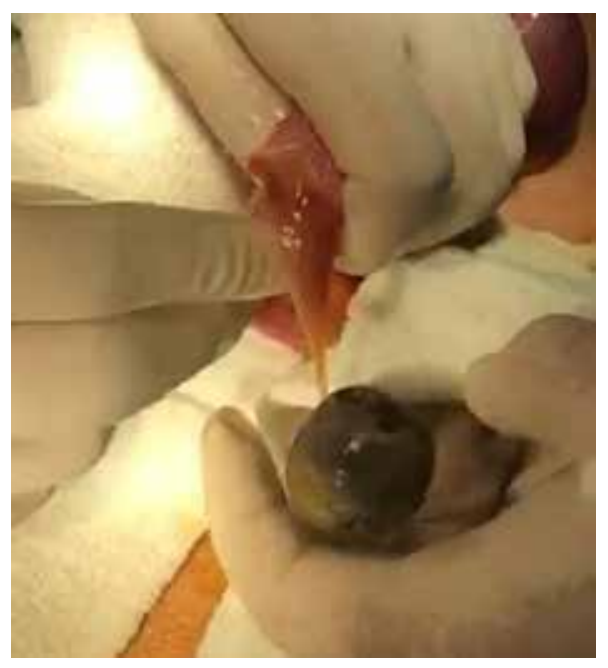

Figure 5: Stone removal by enterotomy 


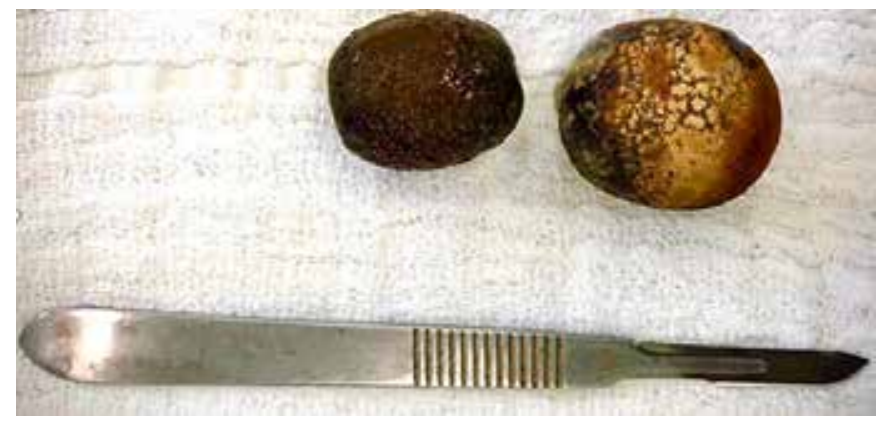

Figure 6: Gallstones.

On the 3rd postoperative day, the patient developed pneumothorax, which was managed with chest drainage, until hospital discharge on the 9th, in good conditions.

\section{DISCUSSION}

The gold standard for imaging diagnosis of the gallstone ileus remains the contrast-enhanced CT of the abdomen ${ }^{7,8}$. However, abdominal US can prove to be a valuable tool in the detection of such cases in geographic regions where access to tomography is limited. Early diagnosis in this case contributed to surgical planning.

Except in cases of cholecystoenteric fistula in which the point of perforation of the gallbladder is in places of difficult access to ultrasound examination, and/or in which the stones have already migrated to the more distal portions of the small intestine, where they may be obscured due to the interposition of intestinal loops and gases, ultrasonography is a useful tool that can contribute to the reduction of the high morbidity and mortality related to this condition ${ }^{9}$.

There are two possible surgical strategies for treating gallstone ileus. The first consists of just alleviating the obstructive process, by removing the stone by enterotomy. The second is to perform cholecystectomy and approach the fistula and its closure. The latter is associated with higher mortality $(16.9 \%)$ when compared to the first $(11.7 \%)^{2}$.

The general conditions of the patient and local inflammatory conditions, which increase technical difficulty, surgical time, and morbidity and mortality, must be considered. Thus, a second elective procedure can be performed, avoiding future biliary complications. The treatment of obstruction remains the basic pillar in the gallstone ileus.

\section{CONCLUSION}

Gallstone ileus is an uncommon condition in the general population, but it contributes to the causes of intestinal obstruction in older patients, with considerable mortality. Clinical suspicion must be high, so that patient management is as effective as possible.

In this case, the diagnostic suspicion was given by ultrasound examination, while the abdominal CT did not identify the obstructive factor.
We emphasize here the importance of abdominal ultrasound as an easily accessible diagnostic tool and the choice of the best surgical approach for each patient.

\section{REFERENCES}

1. Conceiçao SA, Rausch M, Savassi Rocha PR. Tipos especiais de obstruçao intestinal. In Savassi Rocha PR, Souza C: Abdome Agudo, $1^{\mathrm{a}}$ ed, Ed Guanabara Koogan, Rio de Janeiro, 1982

2. Reisner RM, Cohen JR. Gallstone ileus: a review of 1001 reported cases. Am Surg. 1994;60(6): 441-446.

3. Muthukumarasamy G, Venkata SP, Shaikh IA, Somani BK, Ravindran R. Gallstone ileus: surgical strategies and clinical outcome. J Dig Dis. 2008;9(3): 156-161.

4. Halabi WJ, Kang CY, Ketana N, Lafaro KJ, Nguyen VQ, Stamos MJ, Imagawa DK, Demirjian AN. Surgery for gallstone ileus: a nationwide comparison of trends and outcomes. Ann Surg. 2014;259(2): 329-335.

5. Mallipeddi MK, Pappas TN, Shapiro ML, Scarborough JE. Gallstone ileus: revisiting surgical outcomes using National Surgical Quality Improvement Program data. J Surg Res. 2013;184(1): 84-88.

6. O'Brien JW, Webb LA, Evans L, Speakman C, Shaikh I. Gallstone Ileus caused by cholecystocolonic fistula and gallstone impaction in the sigmoid colon: review of the literature and novel surgical treatment with trephine loop colostomy. Case Rep Gastroenterol. 2017;11(1): 95-102.

7. Chang L, Chang M, Chang HM, Chang AI, Chang F. Clinical and radiological diagnosis of gallstone ileus: a mini review. Emerg Radiol. 2018;25(2): 189-196.

8. Yu CY, Lin CC, Shyu RY, Hsieh CB, Wu HS, Tyan YS, Hwang JI, Liou $\mathrm{CH}$, Chang WC, Chen CY. Value of CT in the diagnosis and management of gallstone ileus. World J Gastroenterol. 2005;11(14): 2142-2147.

9. Lasson A, Lorén I, Nilsson A, Nirhov N, Nilsson P. Ultrasonography in gallstone ileus: a diagnostic challenge. Eur J Surg. 1995;161(4): 259-263. 